\title{
Existence Results for Fractional Order Single-Valued and Multi-Valued Problems with Integro-Multistrip-Multipoint Boundary Conditions
}

\author{
Sotiris K. Ntouyas ${ }^{1,2, *,+} \mathbb{D}$, Bashir Ahmad ${ }^{2,+}\left(\mathbb{D}\right.$ and Ahmed Alsaedi ${ }^{2,+}(\mathbb{D}$ \\ 1 Department of Mathematics, University of Ioannina, 45110 Ioannina, Greece \\ 2 Nonlinear Analysis and Applied Mathematics (NAAM)-Research Group, Department of Mathematics, \\ Faculty of Science, King Abdulaziz University, P.P. Box 80203, Jeddah 21589, Saudi Arabia; \\ bashirahmad_qau@yahoo.com (B.A.); aalseadi@hotmail.com (A.A.) \\ * Correspondence: sntouyas@uoi.gr \\ + These authors contributed equally to this work.
}

Received: 28 April 2020; Accepted: 2 July 2020; Published: 5 July 2020

\begin{abstract}
We study the existence of solutions for a new class of boundary value problems of arbitrary order fractional differential equations and inclusions, supplemented with integro-multistripmultipoint boundary conditions. Suitable fixed point theorems are applied to prove some new existence results. The inclusion problem is discussed for convex valued as well as non-convex valued multi-valued map. Examples are also constructed to illustrate the main results. The results presented in this paper are not only new in the given configuration but also provide some interesting special cases.
\end{abstract}

Keywords: differential equation and inclusion; boundary value problem; fractional derivative; fractional integral; fixed point theorem; multi-valued map

\section{Introduction}

Fractional calculus, regarded as a generalization of classical calculus, deals with differential and integral operators of arbitrary non-integer orders. In the last few decades, the tools of fractional calculus considerably improved the mathematical modeling of many real-world phenomena occurring in applied and technical sciences. Examples include co-infection of malaria and HIV/AIDS [1], HIV-immune system with memory [2], chaotic synchronization [3], dynamical networks [4], continuum mechanics [5], financial economics [6], etc. In view of the nonlocal nature of fractional order operators, mathematical models based on such operators are found to be more adequate and informative than their integer order counterparts.

Influenced by application of fractional calculus, there has been shown a significant interest in the investigation of fractional differential equations and inclusions in the recent years. For theory and applications of fractional differential equations, for instance, see the text [7], while the details about Hadamard-type fractional differential equations, inclusions and inequalities can be found in the monograph [8]. In [9], the authors discussed the existence and multiplicity of positive solutions for a singular fractional boundary value problem. Details about variable order integral operators can be found in [10]. In [11], a coupled system of neutral fractional integro-differential equations with infinite delay was studied. For some recent works on fractional differential equations supplemented with multi-point and integral boundary conditions, for instance, see [12-16].

Differential inclusions play a key role in the study of several processes like queueing networks, climate control, optimization of financial issues, for details, see the text [17]. Other applications include synchronization of fractional order systems [18], control systems [19], dynamics of wheeled 
vehicles [20], Navier-Stokes delay differential inclusions [21], etc. Keeping in mind the importance of differential inclusions, several researchers turned to the topic of fractional differential inclusions and obtained a variety of results. In [22,23], the authors investigated semilinear fractional order differential and integro-differential inclusions, respectively. Some existence results for coupled systems of fractional differential inclusions were derived in [24-26], while some recent works on fractional differential inclusions with nonlocal boundary conditions can be found in [27-29]. Oscillation and nonoscillation criteria for Caputo-Hadamard impulsive fractional differential inclusions are presented in [30]. Existence of infinitely many solutions for a fractional differential inclusion with oscillatory potential is discussed in [31]. In a recent paper [32], the existence of solutions for an inclusions problem involving both Caputo and Hadamard fractional derivatives was studied.

Recently, in [33], the authors discussed the existence and uniqueness of solutions for a Caputo type fractional differential equation

$$
{ }^{c} D^{q} x(t)=f(t, x(t)), n-1<q \leq n, t \in J:=[0,1],
$$

equipped with the boundary conditions

$$
\left\{\begin{array}{l}
x(0)=0, x^{\prime}(0)=0, x^{\prime \prime}(0)=0, \ldots, x^{(n-2)}(0)=0, \\
x(1)=a \int_{0}^{\xi} x(s) d s+b \sum_{i=1}^{m-2} \alpha_{i} x\left(\eta_{i}\right), 0<\xi<\eta_{1}<\eta_{2}<\ldots<\eta_{m-2}<1,
\end{array}\right.
$$

where ${ }^{c} D^{q}$ denotes the Caputo fractional derivative of order $q, f: J \times \mathbb{R} \rightarrow \mathbb{R}$ is a continuous function, $a$ and $b$ are real constants and $\alpha_{i}, i=1, \ldots, m-2$, are positive real constants.

In this paper, we are concerned with existence of solutions for a nonlinear fractional differential equation of the form:

$$
D^{\sigma} x(t)=f(t, x(t)), \quad t \in J:=[0,1],
$$

subject to integro-multistrip-multipoint boundary conditions:

$$
x^{(i)}(0)=0, \quad i=0,1,2, \ldots, n-2, \quad \int_{0}^{1} x(s) d s=\sum_{i=2}^{p} \beta_{i-1} \int_{\eta_{i-1}}^{\eta_{i}} x(s) d s+\sum_{j=1}^{q} \gamma_{j} x\left(\rho_{j}\right),
$$

where $D^{\sigma}$ is the standard Riemann-Liouville fractional derivative of order $\sigma$ satisfying $n-1<\sigma \leq n$ with $n \geq 3, f: J \times \mathbb{R} \rightarrow \mathbb{R}$ is a continuous function, $0<\eta_{1}<\eta_{2}<\ldots<\eta_{p}<\rho_{1}<\rho_{2}<\ldots<\rho_{q}<1$, and $\beta_{i}, \gamma_{i}>0, i=2,3, \ldots, p$ with $p, q \in \mathbb{N}$.

Here we remark that the last condition in (4) implies that the sum of the values of the unknown function due to finite many strips and points within the interval $[0,1]$ balances the the average value of the unknown function on the whole interval $[0,1]$.

Existence and uniqueness results are proved for the boundary value problems (3) and (4) by using Krasnoselskii and Banach fixed point theorems.

We also cover the multi-valued case of the problems (3) and (4) by considering the following inclusion problem:

$$
\left\{\begin{array}{l}
D^{\sigma} x(t) \in F(t, x(t)), t \in J:=[0,1] \\
x^{(i)}(0)=0, \quad i=0,1,2, \ldots, n-2, \quad \int_{0}^{1} x(s) d s=\sum_{i=2}^{p} \beta_{i-1} \int_{\eta_{i-1}}^{\eta_{i}} x(s) d s+\sum_{j=1}^{q} \gamma_{j} x\left(\rho_{j}\right),
\end{array}\right.
$$

where $F: J \times \mathbb{R} \rightarrow \mathcal{P}(\mathbb{R})$ is a multi-valued map $(\mathcal{P}(\mathbb{R})$ is the family of all nonempty subsets of $\mathbb{R})$.

The motivation of the present work is to investigate solvability criteria for fractional differential equations and inclusions of arbitrary order subject to a new kind of integro-multistrip-multipoint boundary conditions. The obtained results are not only new in the given configuration but also yield 
several interesting special cases associated with the particular values of the parameters involved in the given problem, including the "zero" average value case (for details, see Conclusions section).

We prove the existence results for the inclusion boundary value problem (5) by using a variety of fixed point theorems sush as Bohnenblust-Karlin fixed point theorem, Martelli fixed point theorem, Leray-Schauder nonlinear alternative, and Covitz-Nadler fixed point theorem.

This paper is organized as follows. In Section 2, we set forth some preliminaries. Section 3 contains existence and uniqueness results for the boundary value problems (3) and (4), while Section 4 deals with the existence of solutions for the inclusion boundary value problem (5). Illustrative examples are also presented.

\section{Preliminaries}

In this section, we recall some definitions from fractional calculus.

Definition 1 ([7]). The Riemann-Liouville fractional integral $I_{a}^{s} f$ of order $s>0$ for a function $f \in$ $L_{1}[a, b],-\infty<a<b<\infty$, existing almost everywhere on $[a, b]$, is defined by

$$
I_{a}^{s} f(t)=\frac{1}{\Gamma(s)} \int_{a}^{t}(t-\tau)^{s-1} f(\tau) d \tau
$$

where $\Gamma$ denotes the Euler gamma function.

Remark 1. Let $f \in L_{p}[a, b], 1 \leq p<\infty$ and $q_{1}, q_{2}>0$. Then the following relation holds almost everywhere on $[a, b]$ :

$$
I_{a}^{q_{1}} I_{a}^{q_{2}} f(t)=I_{a}^{q_{1}+q_{2}} f(t) .
$$

Of course, if $f \in C[a, b]$ or $q_{1}+q_{2}>1$, then the above relation holds for each $t \in[a, b]$.

Definition 2 ([7]). Let $f, f^{(m)} \in L_{1}[a, b]$. Then the Riemann-Liouville fractional derivative $D_{a}^{s} f$ of order $s \in(m-1, m], m \in \mathbb{N}$, existing almost everywhere on $[a, b]$, is defined as

$$
D_{a}^{s} f(t)=\frac{d^{m}}{d t^{m}} I_{a}^{m-s} f(t)=\frac{1}{\Gamma(m-s)} \frac{d^{m}}{d t^{m}} \int_{a}^{t}(t-\tau)^{m-1-s} f(s) d s .
$$

The following lemma is of great importance in the proof of our main results.

Lemma 1. Let $g \in C(J, \mathbb{R})$ and

$$
\rho=\frac{1}{\sigma}\left(1+\sum_{i=2}^{p} \beta_{i-1}\left(\eta_{i}^{\sigma}-\eta_{i-1}^{\sigma}\right)+\sum_{j=1}^{q} \gamma_{j} \rho_{j}^{\sigma-1}\right) \neq 0 .
$$

Then the linear boundary value problem

$$
\begin{gathered}
D^{\sigma} x(t)=g(t), t \in J:=[0,1] \\
x^{(i)}(0)=0, \quad i=0,1,2, \ldots, n-2, \quad \int_{0}^{1} x(s) d s=\sum_{i=2}^{p} \beta_{i-1} \int_{\eta_{i-1}}^{\eta_{i}} x(s) d s+\sum_{j=1}^{q} \gamma_{j} x\left(\rho_{j}\right),
\end{gathered}
$$

has a unique solution given by

$$
x(t)=\frac{1}{\Gamma(\sigma)} \int_{0}^{t}(t-s)^{\sigma-1} g(s) d s+\frac{t^{\sigma-1}}{\rho}\left\{-\frac{1}{\Gamma(\sigma+1)} \int_{0}^{1}(1-s)^{\sigma} g(s) d s\right.
$$




$$
\left.+\sum_{i=2}^{p} \frac{\beta_{i-1}}{\Gamma(\sigma)} \int_{\eta_{i-1}}^{\eta_{i}} \int_{0}^{s}(s-u)^{\sigma-1} g(u) d u d s+\sum_{j=1}^{q} \frac{\gamma_{j}}{\Gamma(\sigma)} \int_{0}^{\rho_{j}}\left(\rho_{j}-s\right)^{\sigma-1} g(s) d s\right\}
$$

Proof. As argued in [7], the general solution of the equation $D^{\sigma} x(t)=g(t), n-1<\sigma<n$ can be written as

$$
x(t)=b_{1} t^{\sigma-1}+b_{2} t^{\sigma-2}+\cdots+b_{n} t^{\sigma-n}+\frac{1}{\Gamma(\sigma)} \int_{0}^{t}(t-s)^{\sigma-1} g(s) d s,
$$

where $b_{i}(i=1,2, \ldots, n)$ are arbitrary constants. Using the conditions $x^{(i)}(0)=0, i=0,1,2, \ldots, n-2$ from (8) into (9), we find that $b_{2}=b_{3}=\cdots=b_{n}=0$ and consequently, (9) takes the form:

$$
x(t)=b_{1} t^{\sigma-1}+\frac{1}{\Gamma(\sigma)} \int_{0}^{t}(t-s)^{\sigma-1} g(s) d s .
$$

Using the last condition of (8) in (10) and solving the resulting equation for $b_{1}$ together with the notation (6), we obtain

$$
\begin{aligned}
b_{1}= & \frac{1}{\rho}\left\{-\frac{1}{\Gamma(\sigma+1)} \int_{0}^{1}(1-s)^{\sigma} g(s) d s+\sum_{i=2}^{p} \frac{\beta_{i-1}}{\Gamma(\sigma)} \int_{\eta_{i-1}}^{\eta_{i}} \int_{0}^{s}(s-u)^{\sigma-1} g(u) d u d s\right. \\
& \left.+\sum_{j=1}^{q} \frac{\gamma_{j}}{\Gamma(\sigma)} \int_{0}^{\rho_{j}}\left(\rho_{j}-s\right)^{\sigma-1} g(s) d s\right\} .
\end{aligned}
$$

Inserting the above values in (9) leads to the solution (9). The converse can be proven by direct computation. The proof is finished.

\section{Existence and Uniqueness Results for Problems (3) and (4)}

By $C(J, \mathbb{R})$ we denote the Banach space of all continuous functions from $J$ into $\mathbb{R}$ endowed with the sup-norm $\|u\|=\sup \{|u(t)|: t \in J\}$. By $L^{1}(J, \mathbb{R})$ we denote the Banach space of Lebesgue integrable functions $u: J \longrightarrow \mathbb{R}$ normed by

$$
\|u\|_{L^{1}}=\int_{0}^{1}|u(t)| d t
$$

In this section we present existence and uniqueness results for the problems (3) and (4). In relation to the problems (3) and (4), we introduce an operator $\mathcal{G}: C(J, \mathbb{R}) \rightarrow C(J, \mathbb{R})$ via Lemma 1 as follows

$$
\begin{aligned}
(\mathcal{G} x)(t)= & \frac{1}{\Gamma(\sigma)} \int_{0}^{t}(t-s)^{\sigma-1} f(s, x(s)) d s+\frac{t^{\sigma-1}}{\rho}\left\{-\frac{1}{\Gamma(\sigma+1)} \int_{0}^{1}(1-s)^{\sigma} f(s, x(s)) d s\right. \\
& +\sum_{i=2}^{p} \frac{\beta_{i-1}}{\Gamma(\sigma)} \int_{\eta_{i-1}}^{\eta_{i}} \int_{0}^{s}(s-u)^{\sigma-1} f(u, x(u)) d u d s \\
& \left.+\sum_{j=1}^{q} \frac{\gamma_{j}}{\Gamma(\sigma)} \int_{0}^{\rho_{j}}\left(\rho_{j}-s\right)^{\sigma-1} f(s, x(s)) d s\right\},
\end{aligned}
$$

such that the the problems (3) and (4) is equivalent to the fixed point problem: $x=\mathcal{G} x$. So the existence of a fixed point of the operator $\mathcal{G}$ will imply the existence of a solution for the problems (3) and (4).

\subsection{Existence Result via Krasnoselskii's Fixed Point Theorem}

Lemma 2. (Krasnoselskii's fixed point theorem [34]). Let $\mathcal{X}$ be a bounded, closed, convex, and nonempty subset of a Banach space $\mathcal{Y}$. Let $\varphi_{1}, \varphi_{2}$ be the operators such that $(i) \varphi_{1} x_{1}+\varphi_{2} x_{2} \in \mathcal{X}$ whenever $x_{1}, x_{2} \in \mathcal{X}$; (ii) $\varphi_{1}$ is compact and continuous; (iii) $\varphi_{2}$ is a contraction mapping. Then there exists $x_{3} \in \mathcal{X}$ such that $x_{3}=\varphi_{1} x_{3}+\varphi_{2} x_{3}$. 
In the sequel, we use the notations:

$$
\Lambda=\frac{1}{\Gamma(\sigma+1)}+\frac{1}{\rho}\left[\frac{1}{\Gamma(\sigma+2)}+\sum_{i=2}^{p}\left|\beta_{i-1}\right| \frac{\eta_{i}^{\sigma+1}-\eta_{i-1}^{\sigma+1}}{\Gamma(\sigma+2)}+\sum_{j=1}^{q}\left|\gamma_{j}\right| \frac{\rho_{j}^{\sigma}}{\Gamma(\sigma+1)}\right]
$$

and

$$
\Lambda_{1}=\Lambda-\frac{1}{\Gamma(\sigma+1)}
$$

Theorem 1. Let $f: J \times \mathbb{R} \rightarrow \mathbb{R}$ be a continuous function satisfying the conditions:

$\left(A_{1}\right)|f(t, x)-f(t, y)| \leq L|x-y|$, for all $t \in J, L>0, x, y \in \mathbb{R}$, with $L<1 / \Lambda_{1}$, where $\Lambda_{1}$ is given by (13);

$\left(A_{2}\right)|f(t, x)| \leq \mu(t)$ for all $(t, x) \in J \times \mathbb{R}, \mu \in C\left(J, \mathbb{R}^{+}\right)$.

Then the boundary value problems (3) and (4) has at least one solution on J.

Proof. We fix $\bar{r} \geq \Lambda\|\mu\|$ and consider the closed ball $B_{\bar{r}}=\{x \in C:\|x\| \leq \bar{r}\}$. We define two operators $\mathcal{G}_{1}$ and $\mathcal{G}_{2}$ on $B_{\bar{r}}$ as

$$
\begin{aligned}
\left(\mathcal{G}_{1} x\right)(t)= & \frac{1}{\Gamma(\sigma)} \int_{0}^{t}(t-s)^{\sigma-1} f(s, x(s)) d s, t \in J, \\
\left(\mathcal{G}_{2} x\right)(t)= & \frac{t^{\sigma-1}}{\rho}\left\{-\frac{1}{\Gamma(\sigma+1)} \int_{0}^{1}(1-s)^{\sigma} f(s, x(s)) d s\right. \\
& +\sum_{i=2}^{p} \frac{\beta_{i-1}}{\Gamma(\sigma)} \int_{\eta_{i-1}}^{\eta_{i}} \int_{0}^{s}(s-u)^{\sigma-1} f(u, x(u)) d u d s \\
& \left.+\sum_{j=1}^{q} \frac{\gamma_{j}}{\Gamma(\sigma)} \int_{0}^{\rho_{j}}\left(\rho_{j}-s\right)^{\sigma-1} f(s, x(s)) d s\right\} .
\end{aligned}
$$

Notice that $\mathcal{G}=\mathcal{G}_{1}+\mathcal{G}_{2}$. For $x, y \in B_{\bar{r}}$, we find that

$$
\begin{aligned}
& \left\|\mathcal{G}_{1} x+\mathcal{G}_{2} y\right\|=\sup _{t \in J}\left|\left(\mathcal{G}_{1} x\right)(t)+\left(\mathcal{G}_{2} y\right)(t)\right| \\
\leq & \|\mu\|\left\{\frac{1}{\Gamma(\sigma)} \int_{0}^{t}(t-s)^{\sigma-1}|f(s, x(s))| d s\right. \\
& +\frac{t^{\sigma-1}}{\rho}\left\{\frac{1}{\Gamma(\sigma+1)} \int_{0}^{1}(1-s)^{\sigma-1}|f(s, y(s))| d s\right. \\
& +\sum_{i=2}^{p} \frac{\left|\beta_{i-1}\right|}{\Gamma(\sigma)} \int_{\eta_{i-1}}^{\eta_{i}} \int_{0}^{s}(s-u)^{\sigma-1}|f(u, y(u))| d u d s \\
& \left.+\sum_{j=1}^{q} \frac{\left|\gamma_{j}\right|}{\Gamma(\sigma)} \int_{0}^{\rho_{j}}\left(\rho_{j}-s\right)^{\sigma-1}|f(s, y(s))| d s\right\} \\
\leq & \|\mu\|\left\{\frac{1}{\Gamma(\sigma+1)}+\frac{1}{\rho}\left[\frac{1}{\Gamma(\sigma+2)}+\sum_{i=2}^{p}\left|\beta_{i-1}\right| \frac{\eta_{i}^{\sigma+1}-\eta_{i-1}^{\sigma+1}}{\Gamma(\sigma+2)}+\sum_{j=1}^{q}\left|\gamma_{j}\right| \frac{\rho_{j}^{\sigma}}{\Gamma(\sigma+1)}\right]\right\} \\
= & \|\mu\| \Lambda \leq \bar{r} .
\end{aligned}
$$


This shows that $\mathcal{G}_{1} x+\mathcal{G}_{2} y \in B_{\bar{r}}$. In the next step we prove that $\mathcal{G}_{2}$ is a contraction mapping. For each $t \in J$ and each $x, y \in C(J, \mathbb{R})$, we have

$$
\begin{aligned}
\left\|\mathcal{G}_{2} x-\mathcal{G}_{2} y\right\| \leq & \sup _{t \in J}\left[\frac { t ^ { \sigma - 1 } } { \rho } \left\{\frac{1}{\Gamma(\sigma+1)} \int_{0}^{1}(1-s)^{\sigma}|f(s, x(s))-f(s, y(s))| d s\right.\right. \\
& +\sum_{i=2}^{p} \frac{\beta_{i-1}}{\Gamma(\sigma)} \int_{\eta_{i-1}}^{\eta_{i}} \int_{0}^{s}(s-u)^{\sigma-1}|f(u, x(u))-f(u, y(u))| d u d s \\
& \left.\left.+\sum_{j=1}^{q} \frac{\gamma_{j}}{\Gamma(\sigma)} \int_{0}^{\rho_{j}}\left(\rho_{j}-s\right)^{\sigma-1}|f(s, x(s))-f(s, y(s))| d s\right\}\right] \\
\leq & \frac{L}{\rho}\left[\frac{1}{\Gamma(\sigma+2)}+\sum_{i=2}^{p}\left|\beta_{i-1}\right| \frac{\eta_{i}^{\sigma+1}-\eta_{i-1}^{\sigma+1}}{\Gamma(\sigma+2)}+\sum_{j=1}^{q}\left|\gamma_{j}\right| \frac{\rho_{j}^{\sigma}}{\Gamma(\sigma+1)}\right]\|x-y\| \\
\leq & L \Lambda_{1}\|x-y\|,
\end{aligned}
$$

which is a contraction mapping by assumption $L \Lambda_{1}<1\left(\Lambda_{1}\right.$ is given by (13)).

The operator $\mathcal{G}_{1}$ is continuous, by the continuity of $f$. Moreover, since

$$
\left\|\mathcal{G}_{1} x\right\| \leq\|\mu\| \frac{1}{\Gamma(q+1)}
$$

the operator $\mathcal{G}_{1}$ is uniformly bounded on $B_{\bar{r}}$.

The compactness of the operator $\mathcal{G}_{1}$ will be proved now. We define $\sup _{(t, x) \in J \times B_{\bar{r}}}|f(t, x)|=\bar{f}$. Consequently, for $0 \leq t_{1}<t_{2} \leq 1$, we have

$$
\begin{aligned}
\left|\mathcal{G}_{1} x\left(t_{2}\right)-\mathcal{G}_{1} x\left(t_{1}\right)\right| \leq & \frac{1}{\Gamma(\sigma)} \mid \int_{0}^{t_{1}}\left[\left(t_{2}-s\right)^{\sigma-1}-\left(t_{1}-s\right)^{\sigma-1}\right] f(s, x(s)) d s \\
& +\int_{t_{1}}^{t_{2}}\left(t_{2}-s\right)^{\sigma-1} f(s, x(s)) d s \mid \\
\leq & \frac{\bar{f}}{\Gamma(\sigma+1)}\left[\left|t_{2}^{\sigma}-t_{1}^{\sigma}\right|+2\left(t_{1}-t_{2}\right)^{\sigma}\right] \rightarrow 0,
\end{aligned}
$$

as $t_{1}-t_{2} \rightarrow 0$, independent of $x$, which means that $\mathcal{G}_{1}$ is relatively compact on $B_{\bar{r}}$. Consequently, by the Arzelá-Ascoli theorem, $\mathcal{G}_{1}$ is compact on $B_{\bar{r}}$. Hence we deduce by the conclusion of Lemma 2 that the problems (3) and (4) has at least one solution on $J$.

\subsection{Uniqueness Result}

Theorem 2. Assume that $f: J \times \mathbb{R} \rightarrow \mathbb{R}$ is a continuous function satisfying the assumption $\left(A_{1}\right)$. Then the problems (3) and (4) has a unique solution on $J$ if $L \Lambda<1$, where $\Lambda$ is given by (12).

Proof. Let us first show that $\mathcal{G} B_{r} \subset B_{r}$, where $\mathcal{G}$ is the operator defined by (11) and $r \geq M \Lambda /(1-L \Lambda)$ with $M=\sup _{t \in J}|f(t, 0)|$. Then, in view of the assumption $\left(A_{3}\right)$, we have

$$
\begin{aligned}
|f(t, x)| & =|f(t, x)-f(t, 0)+f(t, 0)| \leq|f(t, x)-f(t, 0)|+|f(t, 0)| \\
& \leq L\|x\|+M \leq L r+M
\end{aligned}
$$

For any $x \in B_{r}$, we have

$$
\|\mathcal{G} x\|=\sup _{t \in J}|\mathcal{G} x(t)|
$$




$$
\begin{aligned}
\leq & (L r+M)\left\{\frac{1}{\Gamma(\sigma+1)}+\frac{1}{\rho}\left[\frac{1}{\Gamma(\sigma+2)}+\sum_{i=2}^{p}\left|\beta_{i-1}\right| \frac{\eta_{i}^{\sigma+1}-\eta_{i-1}^{\sigma+1}}{\Gamma(\sigma+2)}\right.\right. \\
& \left.\left.+\sum_{j=1}^{q}\left|\gamma_{j}\right| \frac{\rho_{j}^{\sigma}}{\Gamma(\sigma+1)}\right]\right\} \\
= & (L r+M) \Lambda \leq r,
\end{aligned}
$$

which implies that $\mathcal{G} B_{r} \subset B_{r}$. Next, for $x, y \in C(J, \mathbb{R})$ and for each $t \in J$, we obtain

$$
\begin{aligned}
\|\mathcal{G} x-\mathcal{G} y\| \leq & \sup _{t \in J}\left\{\frac{1}{\Gamma(\sigma)} \int_{0}^{t}(t-s)^{\sigma-1}|f(s, x(s))-f(s, y(s))| d s\right. \\
& +\left[\frac { t ^ { \sigma - 1 } } { \rho } \left\{\frac{1}{\Gamma(\sigma+1)} \int_{0}^{1}(1-s)^{\sigma}|f(s, x(s))-f(s, y(s))| d s\right.\right. \\
& +\sum_{i=2}^{p} \frac{\beta_{i-1}}{\Gamma(\sigma)} \int_{\eta_{i-1}}^{\eta_{i}} \int_{0}^{s}(s-u)^{\sigma-1}|f(u, x(u))-f(u, y(u))| d u d s \\
& \left.\left.+\sum_{j=1}^{q} \frac{\gamma_{j}}{\Gamma(\sigma)} \int_{0}^{\rho_{j}}\left(\rho_{j}-s\right)^{\sigma-1}|f(s, x(s))-f(s, y(s))| d s\right\}\right] \\
\leq & L\left\{\frac{1}{\Gamma(\sigma+1)}+\frac{L}{\rho}\left[\frac{1}{\Gamma(\sigma+2)}+\sum_{i=2}^{p}\left|\beta_{i-1}\right| \frac{\eta_{i}^{\sigma+1}-\eta_{i-1}^{\sigma+1}}{\Gamma(\sigma+2)}\right.\right. \\
& \left.\left.+\sum_{j=1}^{q}\left|\gamma_{j}\right| \frac{\rho_{j}^{\sigma}}{\Gamma(\sigma+1)}\right]\right\}\|x-y\| \\
\leq & L \Lambda\|x-y\| .
\end{aligned}
$$

Consequently, the operator $\mathcal{G}$ is a contraction, since $L \Lambda<1$. By Banach contraction mapping principle the operator $\mathcal{G}$ has a unique fixed point, which means that the problems (3) and (4) has a unique solution for on $J$.

\subsection{Examples}

Consider the nonlinear fractional boundary value problem

$$
\begin{gathered}
D^{7 / 2} x(t)=\sqrt{t^{2}+1}+a \tan ^{-1} x(t)+e^{-t} \sin x(t), t \in J:=[0,1], \\
x(0)=0, x^{\prime}(0)=0, \int_{0}^{1} x(s) d s=\frac{3}{4} \int_{1 / 7}^{1 / 6} x(s) d s+\int_{1 / 6}^{1 / 5} x(s) d s+\frac{3}{2} x(1 / 4)+x(1 / 3),
\end{gathered}
$$

where $\sigma=7 / 2, \beta_{1}=3 / 4, \beta_{2}=1, \gamma_{1}=3 / 2, \gamma_{2}=1, \eta_{1}=1 / 7, \eta_{2}=1 / 6, \eta_{3}=1 / 5, \rho_{1}=1 / 4, \rho_{2}=1 / 3$ and $a$ is a positive real number. Using the given values, we find that $\rho \approx 0.318087, \Lambda \approx 0.155011$ and $\Lambda_{1} \approx 0.069039$. It is easy to check that $\mu(t)=\sqrt{t^{2}+1}+\pi a / 2+e^{-t}, L=a+1$ and that $L \Lambda_{1}<1$ holds for $a<13.484567$. As all the conditions of Theorem 1 are satisfied, so the conclusion of Theorem 1 applies to problems (14) and (15). Moreover, $L \Lambda<1$ is satisfied for $a<5.451155$. Thus, by Theorem 2, problems (14) and (15) has a unique solution on $J$.

\section{Existence Results for the Problem (5)}

In the following we use the notations, $\mathcal{P}_{c l}(X)=\{Y \in \mathcal{P}(X): Y$ is closed $\}, \mathcal{P}_{b}(X)=\{Y \in$ $\mathcal{P}(X): Y$ is bounded $\}, \mathcal{P}_{c p}(X)=\{Y \in \mathcal{P}(X): Y$ is compact $\}$, and $\mathcal{P}_{c p, c}(X)=\{Y \in \mathcal{P}(X)$ : $Y$ is compact and convex $\}$. We refer the interested reader to the books by Aubin and Cellina [35], Castaing and Valadier [36], and Deimling [37] for details on multi-valued maps. 
Before stating and proving our main existence results for problem (5), we will give the definition of its solution.

Definition 3. A function $x \in A C^{(n-1)}(J, \mathbb{R})$ is said to be a solution of problem (5) if there exists a function $v \in L^{1}(J, \mathbb{R})$ with $v(t) \in F(t, x)$ a.e. on $J$ such that $x$ satisfies the differential equation $D^{\sigma} x(t)=v(t)$ on $J$ and the boundary condition (4).

\subsection{The Upper Semicontinuous Case}

Consider first the case when $F$ has convex values. Our first result is based on Bohnenblust-Karlin fixed point theorem and closed graph operator theorem, which are stated below.

Lemma 3. (Bohnenblust-Karlin) [38] Let $X$ be a Banach space, $D$ a nonempty subset of $X$, witch is bounded, closed and convex. Suppose $G: J \times \mathbb{R} \rightarrow \mathcal{P}(\mathbb{R})$ is u.s.c. with closed, convex values, and $G(D) \subset D$ and $\overline{G(D)}$ is compact. Then $G$ has a fixed point.

Lemma 4 ([39]). Let $X$ be a separable Banach space. Let $F: J \times X \rightarrow \mathcal{P}_{c p, c}(X)$ be measurable with respect to $t$ for each $y \in X$ and upper semi-continuous with respect to $y$ for almost all $t \in J$ and $S_{F, y} \neq \varnothing$, for any $y \in C(J, X)$, and let $\Theta$ be a linear continuous mapping from $L^{1}(J, X)$ to $C(J, X)$. Then the operator

$$
\Theta \circ S_{F}: C(J, X) \rightarrow \mathcal{P}_{c p, c}(C(J, X)), y \mapsto\left(\Theta \circ S_{F}\right)(y)=\Theta\left(S_{F, y}\right)
$$

is a closed graph operator in $C(J, X) \times C(J, X)$.

Theorem 3. Assume that:

$\left(H_{1}\right) \quad F: J \times \mathbb{R} \rightarrow \mathcal{P}_{c p, c}(\mathbb{R})$ is Carathéodory, i.e.:

(i) $t \longmapsto F(t, y)$ is measurable for each $y \in \mathbb{R}$;

(ii) $y \longmapsto F(t, y)$ is u.s.c. for almost all $t \in J$;

$\left(H_{2}\right)$ for each $r>0$, there exists $\phi_{r} \in L^{1}\left(J, \mathbb{R}^{+}\right)$such that

$$
\|F(t, x)\|=\sup \left\{|v|: v \in F(t, x\} \leq \phi_{r}(t)\right.
$$

for all $x \in \mathbb{R}$ with $\|x\| \leq$ and for a.e. $t \in J$,

$$
\liminf _{r \rightarrow \infty} \frac{1}{r} \int_{0}^{1} \phi_{r}(t) d t=\mu .
$$

Then, if

$$
\left\{\frac{1}{\Gamma(\sigma)}+\frac{1}{\rho}\left[\frac{1}{\Gamma(\sigma+1)}+\frac{1}{\Gamma(\sigma+1)} \sum_{i=2}^{p}\left|\beta_{i-1}\right|+\sum_{j=1}^{q}\left|\gamma_{j}\right| \frac{\rho_{i}^{\sigma-1}}{\Gamma(\sigma)}\right]\right\} \mu<1,
$$

the boundary problem (5) has at least one solution on J. 
Proof. We consider the multi-valued map: $N: C(J, \mathbb{R}) \rightarrow \mathcal{P}(C(J, \mathbb{R}))$ defined by

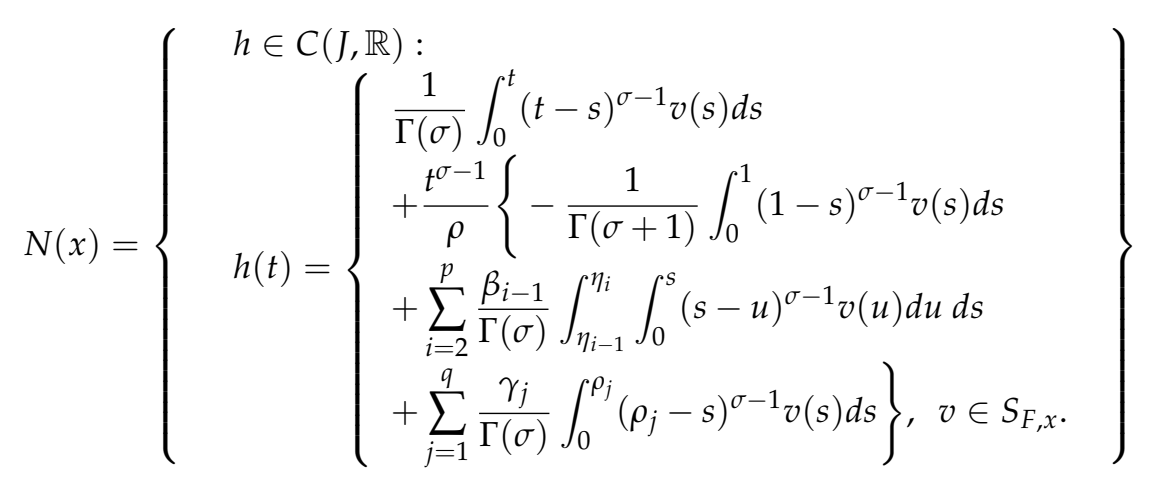

Note that problem (5) is transformed into a fixed point problem, for which the fixed points of $N$ are solutions of problem (5). We will show that the operator $N$ satisfies all condition of Lemma 3. The proof is constructed in several steps.

Step 1. $N(x)$ is convex for each $x \in C(J, \mathbb{R})$.

Let $h_{1}, h_{2}$ in $N(x)$. Then, there exist $v_{1}, v_{2} \in S_{F, x}$ such that

$$
\begin{aligned}
h_{i}(t)= & \frac{1}{\Gamma(\sigma)} \int_{0}^{t}(t-s)^{\sigma-1} v_{i}(s) d s+\frac{t^{\sigma-1}}{\rho}\left\{-\frac{1}{\Gamma(\sigma+1)} \int_{0}^{1}(1-s)^{\sigma-1} v_{i}(s) d s\right. \\
& +\sum_{i=2}^{p} \frac{\beta_{i-1}}{\Gamma(\sigma)} \int_{\eta_{i-1}}^{\eta_{i}} \int_{0}^{s}(s-u)^{\sigma-1} v_{i}(u) d u d s \\
& \left.+\sum_{j=1}^{q} \frac{\gamma_{j}}{\Gamma(\sigma)} \int_{0}^{\rho_{j}}\left(\rho_{j}-s\right)^{\sigma-1} v_{i}(s) d s\right\}, i=1,2, \quad t \in J .
\end{aligned}
$$

Let $0 \leq \theta \leq 1$. Then for each $t \in J$, we have

$$
\begin{aligned}
{\left[\theta h_{1}+(1-\theta) h_{2}\right](t)=} & \frac{1}{\Gamma(\sigma)} \int_{0}^{t}(t-s)^{\sigma-1}\left[\theta v_{1}(s)+(1-\theta) v_{2}(s)\right] d s \\
& +\frac{t^{\sigma-1}}{\rho}\left\{-\frac{1}{\Gamma(\sigma+1)} \int_{0}^{1}(1-s)^{\sigma-1}\left[\theta v_{1}(s)+(1-\theta) v_{2}(s)\right] d s\right. \\
& +\sum_{i=2}^{p} \frac{\beta_{i-1}}{\Gamma(\sigma)} \int_{\eta_{i-1}}^{\eta_{i}} \int_{0}^{s}(s-u)^{\sigma-1}\left[\theta v_{1}(s)+(1-\theta) v_{2}(u)\right] d u d s \\
& \left.+\sum_{j=1}^{q} \frac{\gamma_{j}}{\Gamma(\sigma)} \int_{0}^{\rho_{j}}\left(\rho_{j}-s\right)^{\sigma-1}\left[\theta v_{1}(s)+(1-\theta) v_{2}(s)\right] d s\right\} .
\end{aligned}
$$

Since $F$ has convex values, that is, $S_{F, x}$ is convex, we have

$$
\theta h_{1}+(1-\theta) h_{2} \in N(x) .
$$

Step 2. $N(x)$ maps bounded sets into bounded sets in $C(J, \mathbb{R})$.

Let $B_{r}=\{x \in C(J, \mathbb{R}):\|x\| \leq r\}, r>0$ be a bounded ball in $C(J, \mathbb{R})$. We shall prove that there exists a positive number $r^{\prime}$ such that $N\left(B_{r^{\prime}}\right) \subseteq B_{r}$. If this is not true, for each $r>0$, there exists a function $x_{r}(\cdot) \in B_{r}$, with $\left\|N\left(x_{r}\right)\right\|>r$ for some $t \in J$ and 
Fractal Tract. 2020, 4, 31

10 of 20

$$
\begin{aligned}
h_{r}(t)= & \frac{1}{\Gamma(\sigma)} \int_{0}^{t}(t-s)^{\sigma-1} v_{r}(s) d s+\frac{t^{\sigma-1}}{\rho}\left\{-\frac{1}{\Gamma(\sigma+1)} \int_{0}^{1}(1-s)^{\sigma-1} v_{r}(s) d s\right. \\
& +\sum_{i=2}^{p} \frac{\beta_{i-1}}{\Gamma(\sigma)} \int_{\eta_{i-1}}^{\eta_{i}} \int_{0}^{s}(s-u)^{\sigma-1} v_{r}(u) d u d s \\
& \left.+\sum_{j=1}^{q} \frac{\gamma_{j}}{\Gamma(\sigma)} \int_{0}^{\rho_{j}}\left(\rho_{j}-s\right)^{\sigma-1} v_{r}(s) d s\right\}
\end{aligned}
$$

for some $v_{r} \in S_{F, x_{r}}$. However, on the other hand, we have:

$$
\begin{aligned}
r< & \left\|N\left(x_{r}\right)\right\| \\
\leq & \frac{1}{\Gamma(\sigma)} \int_{0}^{t}(t-s)^{\sigma-1}\left|v_{r}(s)\right| d s+\frac{t^{\sigma-1}}{\rho}\left\{\frac{1}{\Gamma(\sigma+1)} \int_{0}^{1}(1-s)^{\sigma-1}\left|v_{r}(s)\right| d s\right. \\
& \left.+\sum_{i=2}^{p} \frac{\left|\beta_{i-1}\right|}{\Gamma(\sigma)} \int_{\eta_{i-1}}^{\eta_{i}} \int_{0}^{s}(s-u)^{\sigma-1}\left|v_{r}(u)\right| d u d s+\sum_{j=1}^{q} \frac{\left|\gamma_{j}\right|}{\Gamma(\sigma)} \int_{0}^{\rho_{j}}\left(\rho_{j}-s\right)^{\sigma-1}\left|v_{r}(s)\right| d s\right\} \\
\leq & \left\{\frac{1}{\Gamma(\sigma)}+\frac{1}{\rho}\left[\frac{1}{\Gamma(\sigma+1)}+\frac{(p-1)}{\Gamma(\sigma+1)} \sum_{i=2}^{p}\left|\beta_{i-1}\right|+\sum_{j=1}^{q}\left|\gamma_{j}\right| \frac{\rho_{i}^{\sigma-1}}{\Gamma(\sigma)}\right]\right\} \int_{0}^{1} \phi_{r}(s) d s .
\end{aligned}
$$

Dividing both sides by $r$ and taking the lower limit as $r \rightarrow \infty$, we get:

$$
1 \leq \mu\left\{\frac{1}{\Gamma(\sigma)}+\frac{1}{\rho}\left[\frac{1}{\Gamma(\sigma+1)}+\frac{1}{\Gamma(\sigma+1)} \sum_{i=2}^{p}\left|\beta_{i-1}\right|+\sum_{j=1}^{q} \gamma_{j} \mid \frac{\rho_{i}^{\sigma-1}}{\Gamma(\sigma)}\right]\right\}
$$

which contradicts (17). Hence there exists a positive number $r$ such that $N\left(B_{r}\right) \subseteq B_{r}$.

Step 3. $N(x)$ maps bounded sets into equicontinuous sets of $C(J, \mathbb{R})$.

Let $x \in B_{r}$ and $h \in N(x)$. Then there exists $v \in S_{F, x}$ such that

$$
\begin{aligned}
h(t)= & \frac{1}{\Gamma(\sigma)} \int_{0}^{t}(t-s)^{\sigma-1} v(s) d s+\frac{t^{\sigma-1}}{\rho}\left\{-\frac{1}{\Gamma(\sigma+1)} \int_{0}^{1}(1-s)^{\sigma-1} v(s) d s\right. \\
& \left.+\sum_{i=2}^{p} \frac{\beta_{i-1}}{\Gamma(\sigma)} \int_{\eta_{i-1}}^{\eta_{i}} \int_{0}^{s}(s-u)^{\sigma-1} v(u) d u d s+\sum_{j=1}^{q} \frac{\gamma_{j}}{\Gamma(\sigma)} \int_{0}^{\rho_{j}}\left(\rho_{j}-s\right)^{\sigma-1} v(s) d s\right\}, \quad t \in J .
\end{aligned}
$$

Let $t_{1}, t_{2} \in J, t_{1}<t_{2}$. Thus

$$
\begin{aligned}
& \left|h\left(t_{2}\right)-h\left(t_{1}\right)\right| \\
\leq & \frac{1}{\Gamma(\sigma)}\left|\int_{0}^{t_{1}}\left[\left(t_{2}-s\right)^{\sigma-1}-\left(t_{1}-s\right)^{\sigma-1}\right] v(s) d s+\int_{t_{1}}^{t_{2}}\left(t_{2}-s\right)^{\sigma-1} v(s) d s\right| \\
& +\frac{\left|t_{2}^{\sigma-1}-t_{1}^{\sigma-1}\right|}{\rho}\left\{\frac{1}{\Gamma(\sigma+1)} \int_{0}^{1}(1-s)^{\sigma-1}|v(s)| d s\right. \\
& \left.+\sum_{i=2}^{p} \frac{\left|\beta_{i-1}\right|}{\Gamma(\sigma)} \int_{\eta_{i-1}}^{\eta_{i}} \int_{0}^{s}(s-u)^{\sigma-1}|v(u)| d u d s+\sum_{j=1}^{q} \frac{\left|\gamma_{j}\right|}{\Gamma(\sigma)} \int_{0}^{\rho_{j}}\left(\rho_{j}-s\right)^{\sigma-1}|v(s)| d s\right\} \\
\leq & \frac{1}{\Gamma(\sigma)}\left|\int_{0}^{t_{1}}\left[\left(t_{2}-s\right)^{\sigma-1}-\left(t_{1}-s\right)^{\sigma-1}\right] \phi_{r}(s) d s+\int_{t_{1}}^{t_{2}}\left(t_{2}-s\right)^{\sigma-1} \phi_{r}(s) d s\right|
\end{aligned}
$$




$$
\begin{aligned}
& +\frac{\left|t_{2}^{\sigma-1}-t_{1}^{\sigma-1}\right|}{\rho}\left\{\frac{1}{\Gamma(\sigma+1)} \int_{0}^{1}(1-s)^{\sigma-1} \phi_{r}(s) d s\right. \\
& \left.+\sum_{i=2}^{p} \frac{\left|\beta_{i-1}\right|}{\Gamma(\sigma)} \int_{\eta_{i-1}}^{\eta_{i}} \int_{0}^{s}(s-u)^{\sigma-1} \phi_{r}(u) d u d s+\sum_{j=1}^{q} \frac{\left|\gamma_{j}\right|}{\Gamma(\sigma)} \int_{0}^{\rho_{j}}\left(\rho_{j}-s\right)^{\sigma-1} \phi_{r}(s) d s\right\}
\end{aligned}
$$

which tends to zero independently of $x \in B_{r}$ as $t_{1} \rightarrow t_{2}$. As a consequence of Steps 1-3, by using Arzelá-Ascoli theorem, the operator $N: C(J, \mathbb{R}) \rightarrow \mathcal{P}(C(J, \mathbb{R}))$ is completely continuous.

Step 4. $N(x)$ is closed for each $x \in C(J, \mathbb{R})$.

Let $\left\{u_{n}\right\}_{n \geq 0} \in N(x)$ be such that $u_{n} \rightarrow u(n \rightarrow \infty)$ in $C(J, \mathbb{R})$. Then $u \in C(J, \mathbb{R})$ and there exists $v_{n} \in S_{F, x_{n}}$ such that

$$
\begin{aligned}
u_{n}(t)= & \frac{1}{\Gamma(\sigma)} \int_{0}^{t}(t-s)^{\sigma-1} v_{n}(s) d s+\frac{t^{\sigma-1}}{\rho}\left\{-\frac{1}{\Gamma(\sigma+1)} \int_{0}^{1}(1-s)^{\sigma-1} v_{n}(s) d s\right. \\
& \left.+\sum_{i=2}^{p} \frac{\beta_{i-1}}{\Gamma(\sigma)} \int_{\eta_{i-1}}^{\eta_{i}} \int_{0}^{s}(s-u)^{\sigma-1} v_{n}(u) d u d s+\sum_{j=1}^{q} \frac{\gamma_{j}}{\Gamma(\sigma)} \int_{0}^{\rho_{j}}\left(\rho_{j}-s\right)^{\sigma-1} v_{n}(s) d s\right\}, t \in J .
\end{aligned}
$$

Since $F$ has compact values, we can pass onto a subsequence (if necessary) to obtain that $v_{n}$ converges to $v$ in $L^{1}(J, \mathbb{R})$. Thus $v \in S_{F, x}$ and we have

$$
\begin{aligned}
& u_{n}(t) \rightarrow v(t) \\
= & \frac{1}{\Gamma(\sigma)} \int_{0}^{t}(t-s)^{\sigma-1} v(s) d s+\frac{t^{\sigma-1}}{\rho}\left\{-\frac{1}{\Gamma(\sigma+1)} \int_{0}^{1}(1-s)^{\sigma-1} v(s) d s\right. \\
& \left.+\sum_{i=2}^{p} \frac{\beta_{i-1}}{\Gamma(\sigma)} \int_{\eta_{i-1}}^{\eta_{i}} \int_{0}^{s}(s-u)^{\sigma-1} v(u) d u d s+\sum_{j=1}^{q} \frac{\gamma_{j}}{\Gamma(\sigma)} \int_{0}^{\rho_{j}}\left(\rho_{j}-s\right)^{\sigma-1} v(s) d s\right\}, t \in J .
\end{aligned}
$$

Hence, $u \in N(x)$.

Next we show that the operator $N$ is upper semicontinuous. In order to do so, by Proposition 1.2 in [37], we know that it will be upper semicontinuous has a closed graph. This will be shown in the next step.

Step 5. $N$ has a closed graph.

Let $x_{n} \rightarrow x_{*}, h_{n} \in N\left(x_{n}\right)$ and $h_{n} \rightarrow h_{*}$. We will show that $h_{*} \in N\left(x_{*}\right)$. Now $h_{n} \in N\left(x_{n}\right)$ implies that there exists $v_{n} \in S_{F, x_{n}}$ such that

$$
\begin{aligned}
h_{n}(t)= & \frac{1}{\Gamma(\sigma)} \int_{0}^{t}(t-s)^{\sigma-1} v_{n}(s) d s+\frac{t^{\sigma-1}}{\rho}\left\{-\frac{1}{\Gamma(\sigma+1)} \int_{0}^{1}(1-s)^{\sigma-1} v_{n}(s) d s\right. \\
& \left.+\sum_{i=2}^{p} \frac{\beta_{i-1}}{\Gamma(\sigma)} \int_{\eta_{i-1}}^{\eta_{i}} \int_{0}^{s}(s-u)^{\sigma-1} v_{n}(u) d u d s+\sum_{j=1}^{q} \frac{\gamma_{j}}{\Gamma(\sigma)} \int_{0}^{\rho_{j}}\left(\rho_{j}-s\right)^{\sigma-1} v_{n}(s) d s\right\}, t \in J .
\end{aligned}
$$

We must show that there exists $v_{*} \in S_{F, x_{*}}$ such that for each $t \in J$,

$$
h_{*}(t)=\frac{1}{\Gamma(\sigma)} \int_{0}^{t}(t-s)^{\sigma-1} v_{*}(s) d s+\frac{t^{\sigma-1}}{\rho}\left\{-\frac{1}{\Gamma(\sigma+1)} \int_{0}^{1}(1-s)^{\sigma-1} v_{*}(s) d s\right.
$$




$$
\left.+\sum_{i=2}^{p} \frac{\beta_{i-1}}{\Gamma(\sigma)} \int_{\eta_{i-1}}^{\eta_{i}} \int_{0}^{s}(s-u)^{\sigma-1} v_{*}(u) d u d s+\sum_{j=1}^{q} \frac{\gamma_{j}}{\Gamma(\sigma)} \int_{0}^{\rho_{j}}\left(\rho_{j}-s\right)^{\sigma-1} v_{*}(s) d s\right\}
$$

Consider the continuous linear operator $\Theta: L^{1}(J, \mathbb{R}) \rightarrow C(J)$ by

$$
\begin{aligned}
v \rightarrow \Theta(v)(t)= & \frac{1}{\Gamma(\sigma)} \int_{0}^{t}(t-s)^{\sigma-1} v_{n}(s) d s+\frac{t^{\sigma-1}}{\rho}\left\{-\frac{1}{\Gamma(\sigma+1)} \int_{0}^{1}(1-s)^{\sigma-1} v_{n}(s) d s\right. \\
& +\sum_{i=2}^{p} \frac{\beta_{i-1}}{\Gamma(\sigma)} \int_{\eta_{i-1}}^{\eta_{i}} \int_{0}^{s}(s-u)^{\sigma-1} v_{n}(u) d u d s \\
& \left.+\sum_{j=1}^{q} \frac{\gamma_{j}}{\Gamma(\sigma)} \int_{0}^{\rho_{j}}\left(\rho_{j}-s\right)^{\sigma-1} v_{n}(s) d s\right\} .
\end{aligned}
$$

Observe that $\left\|h_{n}(t)-h_{*}(t)\right\| \rightarrow 0$ as $n \rightarrow \infty$, and thus, it follows from a closed graph Lemma ([39]), that $\Theta \circ S_{F, x}$ is a closed graph operator. Moreover, we have

$$
h_{n} \in \Theta\left(S_{F, x_{n}}\right) .
$$

Since $x_{n} \rightarrow x_{*}$, the closed graph Lemma ([39]) implies that

$$
\begin{aligned}
h_{*}(t)= & \frac{1}{\Gamma(\sigma)} \int_{0}^{t}(t-s)^{\sigma-1} v_{*}(s) d s+\frac{t^{\sigma-1}}{\rho}\left\{-\frac{1}{\Gamma(\sigma+1)} \int_{0}^{1}(1-s)^{\sigma-1} v_{*}(s) d s\right. \\
& \left.+\sum_{i=2}^{p} \frac{\beta_{i-1}}{\Gamma(\sigma)} \int_{\eta_{i-1}}^{\eta_{i}} \int_{0}^{s}(s-u)^{\sigma-1} v_{*}(u) d u d s+\sum_{j=1}^{q} \frac{\gamma_{j}}{\Gamma(\sigma)} \int_{0}^{\rho_{j}}\left(\rho_{j}-s\right)^{\sigma-1} v_{*}(s) d s\right\},
\end{aligned}
$$

for some $v_{*} \in S_{F, x_{*}}$

Hence, we conclude that $N$ is a compact multivalued map, upper semicontinuous with convex closed values. Consequently by Lemma $3, N$ has a fixed point which is a solution of the boundary problem (5). This completes the proof.

Next, we give an existence result based upon the following form of fixed point theorem which is applicable to completely continuous operators.

Lemma 5. [40] Let $E$ a Banach space, and $T: E \rightarrow \mathcal{P}_{b, c l, c}(E)$ be a completely continuous multi-valued map. If the set $\mathcal{Z}=\{x \in E: \lambda x \in T(x), \lambda>1\}$ is bounded, then $T$ has a fixed point.

Theorem 4. Assume that the following hypotheses hold:

$\left(H_{3}\right) F: J \times \mathbb{R} \rightarrow \mathcal{P}_{b, c l, c}(\mathbb{R})$ is a Carathéodory multi-valued map;

$\left(H_{4}\right)$ there exists a function $h \in C(J, \mathbb{R})$ such that

$$
\|F(t, x)\| \leq h(t), \text { for a.e. } t \in J \text { and each } x \in \mathbb{R} .
$$

Then problem (5) has at least one solution on J.

Proof. Consider $N$ defined in the proof of Theorem 3. As in Theorem 3, we can show that $N$ is convex and completely continuous. It remains to show that the set

$$
\mathcal{E}=\{x \in C(J, \mathbb{R}): \lambda x \in N(x), \lambda>1\}
$$


is bounded. Let $x \in \mathcal{E}$, then $\lambda x \in N(x)$ for some $\lambda>1$ and there exists a function $v \in S_{F, x}$ such that

$$
\begin{aligned}
x(t)= & \frac{1}{\lambda} \frac{1}{\Gamma(\sigma)} \int_{0}^{t}(t-s)^{\sigma-1} v(s) d s+\frac{1}{\lambda} \frac{t^{\sigma-1}}{\rho}\left\{-\frac{1}{\Gamma(\sigma+1)} \int_{0}^{1}(1-s)^{\sigma-1} v(s) d s\right. \\
& \left.+\sum_{i=2}^{p} \frac{\beta_{i-1}}{\Gamma(\sigma)} \int_{\eta_{i-1}}^{\eta_{i}} \int_{0}^{s}(s-u)^{\sigma-1} v(u) d u d s+\sum_{j=1}^{q} \frac{\gamma_{j}}{\Gamma(\sigma)} \int_{0}^{\rho_{j}}\left(\rho_{j}-s\right)^{\sigma-1} v(s) d s\right\} .
\end{aligned}
$$

For each $t \in J$, we have

$$
\begin{aligned}
|x(t)| \leq & \frac{1}{\Gamma(\sigma)} \int_{0}^{t}(t-s)^{\sigma-1}|v(s)| d s+\frac{t^{\sigma-1}}{\rho}\left\{\frac{1}{\Gamma(\sigma+1)} \int_{0}^{1}(1-s)^{\sigma-1}|v(s)| d s\right. \\
& \left.+\sum_{i=2}^{p} \frac{\left|\beta_{i-1}\right|}{\Gamma(\sigma)} \int_{\eta_{i-1}}^{\eta_{i}} \int_{0}^{s}(s-u)^{\sigma-1}|v(u)| d u d s+\sum_{j=1}^{q} \frac{\left|\gamma_{j}\right|}{\Gamma(\sigma)} \int_{0}^{\rho_{j}}\left(\rho_{j}-s\right)^{\sigma-1}|v(s)| d s\right\} \\
\leq & \left\{\frac{1}{\Gamma(\sigma+1)}+\frac{1}{\rho}\left[\frac{1}{\Gamma(\sigma+2)}+\sum_{i=2}^{p}\left|\beta_{i-1}\right| \frac{\eta_{i}^{\sigma+1}-\eta_{i-1}^{\sigma+1}}{\Gamma(\sigma+2)}+\sum_{j=1}^{q}\left|\gamma_{j}\right| \frac{\rho_{j}^{\sigma}}{\Gamma(\sigma+1)}\right]\right\}\|h\| .
\end{aligned}
$$

Taking the supremum for $t \in J$, we get

$$
\|x\| \leq\left\{\frac{1}{\Gamma(\sigma+1)}+\frac{1}{\rho}\left[\frac{1}{\Gamma(\sigma+2)}+\sum_{i=2}^{p}\left|\beta_{i-1}\right| \frac{\eta_{i}^{\sigma+1}-\eta_{i-1}^{\sigma+1}}{\Gamma(\sigma+2)}+\sum_{j=1}^{q}\left|\gamma_{j}\right| \frac{\rho_{j}^{\sigma}}{\Gamma(\sigma+1)}\right]\right\}\|h\|<\infty .
$$

Hence the set $\mathcal{E}$ is bounded. As a consequence of Lemma 5 we deduce that $N$ has at least one fixed point which implies that problem (5) has a solution on $J$.

Our final existence result in this subsection is based on Leray-Schauder nonlinear alternative [41].

Theorem 5. Assume that $\left(H_{1}\right)$ holds. In addition we suppose that:

$\left(H_{5}\right)$ there exist a nondecreasing continuous function $\Psi:[0, \infty) \rightarrow(0, \infty)$ and a continuous function $\phi \in C\left(J, \mathbb{R}^{+}\right)$such that $\|F(t, x)\|_{\mathcal{P}}:=\sup \{|y|: y \in F(t, x)\} \leq \phi(t) \Psi(\|x\|)$ for each $(t, x) \in J \times \mathbb{R} ;$

$\left(H_{6}\right)$ there exists a constant $M>0$ such that

$$
\frac{M}{\Psi(M)\|\phi\| \Lambda}>1
$$

Then, the boundary value problem (5) has at least one solution on J.

Proof. Let $x \in \lambda N(x)$ for some $\lambda \in(0,1)$, where the operator $N$ is defined in the proof of Theorem 3 . We show there exists an open set $\mathcal{W} \subseteq C(J, \mathbb{R})$ with $x \notin N(x)$ for any $\lambda \in(0,1)$ and all $x \in \partial \mathcal{W}$. Let $\lambda \in(0,1)$ and $x \in \lambda N(x)$. Then there exists $v \in L^{1}(J, \mathbb{R})$ with $v \in S_{F, x}$ such that, for $t \in J$, we have

$$
\begin{aligned}
x(t)= & \lambda \frac{1}{\Gamma(\sigma)} \int_{0}^{t}(t-s)^{\sigma-1} v(s) d s+\lambda \frac{t^{\sigma-1}}{\rho}\left\{-\frac{1}{\Gamma(\sigma+1)} \int_{0}^{1}(1-s)^{\sigma-1} v(s) d s\right. \\
& \left.+\sum_{i=2}^{p} \frac{\beta_{i-1}}{\Gamma(\sigma)} \int_{\eta_{i-1}}^{\eta_{i}} \int_{0}^{s}(s-u)^{\sigma-1} v(u) d u d s+\sum_{j=1}^{q} \frac{\gamma_{j}}{\Gamma(\sigma)} \int_{0}^{\rho_{j}}\left(\rho_{j}-s\right)^{\sigma-1} v(s) d s\right\} .
\end{aligned}
$$

In view of $\left(H_{5}\right)$, we have for each $t \in J$,

$$
|x(t)|
$$




$$
\begin{aligned}
\leq & \frac{1}{\Gamma(\sigma)} \int_{0}^{t}(t-s)^{\sigma-1}|v(s)| d s+\frac{t^{\sigma-1}}{\rho}\left\{\frac{1}{\Gamma(\sigma+1)} \int_{0}^{1}(1-s)^{\sigma-1}|v(s)| d s\right. \\
& \left.+\sum_{i=2}^{p} \frac{\left|\beta_{i-1}\right|}{\Gamma(\sigma)} \int_{\eta_{i-1}}^{\eta_{i}} \int_{0}^{s}(s-u)^{\sigma-1}|v(u)| d u d s+\sum_{j=1}^{q} \frac{\left|\gamma_{j}\right|}{\Gamma(\sigma)} \int_{0}^{\rho_{j}}\left(\rho_{j}-s\right)^{\sigma-1}|v(s)| d s\right\} \\
\leq & \left\{\frac{1}{\Gamma(\sigma+1)}+\frac{1}{\rho}\left[\frac{1}{\Gamma(\sigma+2)}+\sum_{i=2}^{p}\left|\beta_{i-1}\right| \frac{\eta_{i}^{\sigma+1}-\eta_{i-1}^{\sigma+1}}{\Gamma(\sigma+2)}+\sum_{j=1}^{q}\left|\gamma_{j}\right| \frac{\rho_{j}^{\sigma}}{\Gamma(\sigma+1)}\right]\right\}\|\phi\| \Psi(\|x\|) .
\end{aligned}
$$

Consequently, we have

$$
\frac{\|x\|}{\Psi(\|x\|)\|\phi\| \Lambda} \leq 1
$$

In view of $\left(H_{6}\right)$, there exists $M$ such that $\|x\| \neq M$. Let us set

$$
\mathcal{W}=\{x \in C(J, \mathbb{R}):\|x\|<M\} .
$$

Proceeding as in the proof of Theorem 3, one can show that the operator $N: \overline{\mathcal{W}} \rightarrow \mathcal{P}(C(J, \mathbb{R}))$ is a compact, upper semicontinuous multi-valued map with convex closed values. Note that there is no $x \in \partial \mathcal{W}$ such that $x \in \lambda N(x)$ for some $\lambda \in(0,1)$, from the choice of $\mathcal{W}$. Therefore, by the nonlinear alternative of Leray-Schauder type ([41]), we deduce that $N$ has a fixed point $x \in \overline{\mathcal{W}}$. Obviously this fixed point is a solution of the boundary value problem (5). The proof is completed.

\subsection{The Lower Semicontinuous Case}

In this subsection we study the lower semicontinuous case, when $F$ is not necessarily convex valued. Our strategy is to apply the nonlinear alternative of Leray-Schauder type together with the selection theorem of Bressan and Colombo [42] for lower semi-continuous maps with decomposable values. The needed preliminaries can be found in [35].

Theorem 6. Assume that $\left(H_{5}\right),\left(H_{6}\right)$ hold. Moreover, we suppose that:

$\left(H_{7}\right) F: J \times \mathbb{R} \rightarrow \mathcal{P}(\mathbb{R})$ is a nonempty compact-valued multivalued map such that $x \longmapsto F(t, x)$ is lower semicontinuous for each $t \in J$ and $(t, x) \longmapsto F(t, x)$ is $\mathcal{L} \otimes \mathcal{B}$ measurable.

Then the boundary value problem (5) has at least one solution on $J$.

Proof. From $\left(H_{5}\right)$ and $\left(H_{7}\right)$, it follows that $F$ is of lower semicontinuous type [43]. Then, by the selection theorem of Bressan and Colombo [42], there exists a continuous function $v: A C^{1}(J, \mathbb{R}) \rightarrow$ $L^{1}(J, \mathbb{R})$ such that $v(x) \in \mathcal{F}(x)$ for all $v \in C(J, \mathbb{R})$, where $\mathcal{F}: C(J \times \mathbb{R}) \rightarrow \mathcal{P}\left(L^{1}(J, \mathbb{R})\right)$ is defined by

$$
\mathcal{F}(v)=\left\{\omega \in L^{1}(J, \mathbb{R}): \omega(t) \in F(t, v(t)) \text { for a.e. } t \in J\right\} .
$$

Consider the problem

$$
\begin{gathered}
D^{\sigma} x(t)=f(x(t)), t \in J:=[0,1] \\
x^{(i)}(0)=0, \quad i=0,1,2, \ldots, n-2, \quad \int_{0}^{1} x(s) d s=\sum_{i=2}^{p} \beta_{i-1} \int_{\eta_{i-1}}^{\eta_{i}} x(s) d s+\sum_{j=1}^{q} \gamma_{j} x\left(\rho_{j}\right) .
\end{gathered}
$$

Observe that $x$ is a solution to the boundary value problem (5) if $x \in A C^{(n-1)}(J, \mathbb{R})$ is a solution of problems (18) and (19). We define an operator $\bar{N}$ as

$$
\bar{N}(x)=\frac{1}{\Gamma(\sigma)} \int_{0}^{t}(t-s)^{\sigma-1} f(x(s)) d s+\frac{t^{\sigma-1}}{\rho}\left\{-\frac{1}{\Gamma(\sigma+1)} \int_{0}^{1}(1-s)^{\sigma-1} f(x(s)) d s\right.
$$




$$
\left.+\sum_{i=2}^{p} \frac{\beta_{i-1}}{\Gamma(\sigma)} \int_{\eta_{i-1}}^{\eta_{i}} \int_{0}^{s}(s-u)^{\sigma-1} f(x(u)) d u d s+\sum_{j=1}^{q} \frac{\gamma_{j}}{\Gamma(\sigma)} \int_{0}^{\rho_{j}}\left(\rho_{j}-s\right)^{\sigma-1} f(x(s)) d s\right\} .
$$

Then the boundary value problems (18) and (19) is transformed into a fixed point problem. The operator $\bar{N}$ is continuous and completely continuous. We omit the rest of the proof, since it is similar to that of Theorem 5. The proof is finished.

\subsection{The Lipschitz Case}

In this subsection, we consider the boundary value problem (5) with a nonconvex valued right hand side. By using a fixed point theorem for multivalued maps due to Covitz and Nadler [44] we prove an existence result.

Lemma 6. (Covitz and Nadler [44]) Let $(X, d)$ be a complete metric space. If $N: X \rightarrow \mathcal{P}_{c l}(X)$ is a contraction, then FixN $\neq \varnothing$.

Theorem 7. Suppose that:

$\left(H_{8}\right) F: J \times \mathbb{R} \rightarrow \mathcal{P}_{c p}(\mathbb{R})$ is such that $F(\cdot, x): J \rightarrow \mathcal{P}_{c p}(\mathbb{R})$ is measurable for each $x \in \mathbb{R}$;

$\left(H_{9}\right) H_{d}(F(t, x), F(t, z)) \leq \mu(t)|x-z|$ for almost all $t \in J$ and $x, z \in \mathbb{R}$ with $\mu \in C\left(J, \mathbb{R}^{+}\right)$and $d(0, F(t, 0)) \leq \mu(t)$ for almost all $t \in J$.

Then, the boundary value problem (5) has at least one solution on J provided that

$$
\Lambda\|\mu\|<1
$$

Proof. Consider the operator $N: C(J, \mathbb{R}) \rightarrow \mathcal{P}(C(J, \mathbb{R}))$ defined at the beginning of the proof of Theorem 3 . Then, the boundary value problem (5) is transformed into a fixed point problem. We apply Lemma 6 in two steps.

Step I. The operator $N$ is closed and nonempty for every $v \in S_{F, x}$.

The set-valued map $F(\cdot, x(\cdot))$ is measurable by the measurable selection theorem (e.g., [36], Theorem III.6) and consequently it admits a measurable selection $v: J \rightarrow \mathbb{R}$. By the assumption $\left(H_{8}\right)$, we have

$$
|v(t)| \leq \mu(t)+\mu(t)|x(t)|,
$$

i.e., $v \in L^{1}(J, \mathbb{R})$ and hence $F$ is integrably bounded. Therefore, $S_{F, x} \neq \varnothing$. Moreover $N(x) \in \mathcal{P}_{c l}(C(J$, $\mathbb{R})$ ) for each $x \in C(J, \mathbb{R})$, as proved in Step 4 of Theorem 3 .

Step II. Now we show that there exists $\theta<1$ such that

$$
H_{d}(N(x), N(\bar{x})) \leq \theta\|x-z\| \text { for each } x, z \in A C^{(n-1)}(J, \mathbb{R}) .
$$

Let $x, \bar{x} \in A C^{(n-1)}(J, \mathbb{R})$ and $h_{1} \in N(x)$. Then there exists $v_{1}(t) \in F(t, x(t))$ such that, for each $t \in J$,

$$
\begin{aligned}
h_{1}(t)= & \frac{1}{\Gamma(\sigma)} \int_{0}^{t}(t-s)^{\sigma-1} v_{1}(s) d s+\frac{t^{\sigma-1}}{\rho}\left\{-\frac{1}{\Gamma(\sigma+1)} \int_{0}^{1}(1-s)^{\sigma-1} v_{1}(s) d s\right. \\
& \left.+\sum_{i=2}^{p} \frac{\beta_{i-1}}{\Gamma(\sigma)} \int_{\eta_{i-1}}^{\eta_{i}} \int_{0}^{s}(s-u)^{\sigma-1} v_{1}(u) d u d s+\sum_{j=1}^{q} \frac{\gamma_{j}}{\Gamma(\sigma)} \int_{0}^{\rho_{j}}\left(\rho_{j}-s\right)^{\sigma-1} v_{1}(s) d s\right\} .
\end{aligned}
$$


By $\left(H_{9}\right)$, we have

$$
H_{d}(F(t, x), F(t, z)) \leq \mu(t)|x(t)-z(t)| .
$$

Thus, there exists $w(t) \in F(t, z(t))$ such that

$$
\left|v_{1}(t)-w\right| \leq \mu(t)|x(t)-z(t)|, \quad t \in J .
$$

Define $U: J \rightarrow \mathcal{P}(\mathbb{R})$ by

$$
U(t)=\left\{w \in \mathbb{R}:\left|v_{1}(t)-w\right| \leq \mu(t)|x(t)-z(t)|\right\} .
$$

The multivalued operator $U(t) \cap F(t, z(t))$ is measurable (Proposition III.4 [36]). Therefore, there exists a function $v_{2}(t)$ which is a measurable selection for $U$. So $v_{2}(t) \in F(t, z(t))$ and $\mid v_{1}(t)-$ $v_{2}(t)|\leq \mu(t)| x(t)-z(t) \mid$ for each $t \in J$.

For each $t \in J$, let us define

$$
\begin{aligned}
h_{2}(t)= & \frac{1}{\Gamma(\sigma)} \int_{0}^{t}(t-s)^{\sigma-1} v_{2}(s) d s+\frac{t^{\sigma-1}}{\rho}\left\{-\frac{1}{\Gamma(\sigma+1)} \int_{0}^{1}(1-s)^{\sigma-1} v_{2}(s) d s\right. \\
& \left.+\sum_{i=2}^{p} \frac{\beta_{i-1}}{\Gamma(\sigma)} \int_{\eta_{i-1}}^{\eta_{i}} \int_{0}^{s}(s-u)^{\sigma-1} v_{2}(u) d u d s+\sum_{j=1}^{q} \frac{\gamma_{j}}{\Gamma(\sigma)} \int_{0}^{\rho_{j}}\left(\rho_{j}-s\right)^{\sigma-1} v_{2}(s) d s\right\} .
\end{aligned}
$$

Then we have

$$
\begin{aligned}
\left|h_{1}(t)-h_{2}(t)\right| \leq & \frac{1}{\Gamma(\sigma)} \int_{0}^{t}(t-s)^{\sigma-1}\left|v_{2}(s)-v_{1}(s)\right| d s \\
& +\frac{t^{\sigma-1}}{\rho}\left\{\frac{1}{\Gamma(\sigma+1)} \int_{0}^{1}(1-s)^{\sigma-1}\left|v_{2}(s)-v_{1}(s)\right| d s\right. \\
& +\sum_{i=2}^{p} \frac{\left|\beta_{i-1}\right|}{\Gamma(\sigma)} \int_{\eta_{i-1}}^{\eta_{i}} \int_{0}^{s}(s-u)^{\sigma-1}\left|v_{2}(u)-v_{1}(u)\right| d u d s \\
& \left.+\sum_{j=1}^{q} \frac{\left|\gamma_{j}\right|}{\Gamma(\sigma)} \int_{0}^{\rho_{j}}\left(\rho_{j}-s\right)^{\sigma-1}\left|v_{2}(s)-v_{1}(s)\right| d s\right\} \\
\leq & \left\{\frac{1}{\Gamma(\sigma+1)}+\frac{1}{\rho}\left[\frac{1}{\Gamma(\sigma+2)}+\sum_{i=2}^{p}\left|\beta_{i-1}\right| \frac{\eta_{i}^{\sigma+1}-\eta_{i-1}^{\sigma+1}}{\Gamma(\sigma+2)}+\sum_{j=1}^{q}\left|\gamma_{j}\right| \frac{\rho_{j}^{\sigma}}{\Gamma(\sigma+1)}\right]\right\} \\
& \times\|\mu\|\|x-z\| .
\end{aligned}
$$

Hence

$$
\left\|h_{1}-h_{2}\right\| \leq \Lambda\|\mu\|\|x-z\| \text {. }
$$

Analogously, interchanging the roles of $x$ and $z$, we obtain

$$
H_{d}(N(x), N(z)) \leq \Lambda\|\mu\|\|x-z\| .
$$

Consequently $N$ is a contraction. Then, by Lemma $6, N$ has a fixed point $x$ which is a solution of the boundary value (5). The proof is completed.

\subsection{Examples}

Consider the nonlinear fractional boundary value problem

$$
D^{7 / 2} x(t) \in F(t, x(t)), \quad t \in J:=[0,1],
$$




$$
x(0)=0, x^{\prime}(0)=0, \int_{0}^{1} x(s) d s=\frac{3}{4} \int_{1 / 7}^{1 / 6} x(s) d s+\int_{1 / 6}^{1 / 5} x(s) d s+\frac{3}{2} x(1 / 4)+x(1 / 3) .
$$

Here $\sigma=7 / 2, \beta_{1}=3 / 4, \beta_{2}=1, \gamma_{1}=3 / 2, \gamma_{2}=1, \eta_{1}=1 / 7, \eta_{2}=1 / 6, \eta_{3}=1 / 5, \rho_{1}=1 / 4, \rho_{2}=$ $1 / 3$. Consequently we can get $\rho=0.318087$.

(a) Let $F: J \times \mathbb{R} \rightarrow \mathcal{P}(\mathbb{R})$ be a multivalued map given by

$$
x \rightarrow F(t, x)=\left[\frac{x^{2} e^{-x^{2}}}{x^{2}+3}, \frac{t|x| \sin |x|}{|x|+1}+1\right] .
$$

For $f \in F$, we have

$$
|f| \leq \max \left(\frac{x^{2} e^{-x^{2}}}{x^{2}+3}, \frac{t|x| \sin |x|}{|x|+1}+1\right) \leq t|x|+1, \quad x \in \mathbb{R} .
$$

Thus $\|F(t, x)\|_{\mathcal{P}}:=\sup \{|y|: y \in F(t, x)\} \leq r t+1=\phi_{r}(t),\|x\| \leq r$, and $\liminf _{r \rightarrow \infty} \frac{1}{r} \int_{0}^{1} \phi_{r}(s) d s=$ $\mu=1 / 2$. Therefore, all the conditions of Theorem 3 are satisfied. So, problems (20) and (21) with $F(t, x)$ given by (22) has at least one solution on $J$ since

$$
\left\{\frac{1}{\Gamma(\sigma)}+\frac{1}{\rho}\left[\frac{1}{\Gamma(\sigma+1)}+\sum_{i=2}^{p}\left|\beta_{i-1}\right| \frac{1}{\Gamma(\sigma+1)}+\sum_{j=1}^{q}\left|\gamma_{j}\right| \frac{\rho_{j}^{\sigma-1}}{\Gamma(\sigma)}\right]\right\} \mu \approx 0.574595<1 .
$$

(b) If $F: J \times \mathbb{R} \rightarrow \mathcal{P}(\mathbb{R})$ is a multivalued map given by

$$
x \rightarrow F(t, x)=\left[\frac{x^{4}}{x^{4}+2}+e^{-x^{2}}+t+2, \frac{|x|}{|x|+1}+t^{2}+\frac{1}{2}\right] .
$$

For $f \in F$, we have

$$
|f| \leq \max \left(\frac{x^{4}}{x^{4}+2}+e^{-x^{2}}+t+2, \frac{|x|}{|x|+1}+t^{2}+\frac{1}{2}\right) \leq 5, \quad x \in \mathbb{R}
$$

Thus $\|F(t, x)\|_{\mathcal{P}}:=\sup \{|y|: y \in F(t, x)\} \leq 5=\phi(t) \Psi(\|x\|), y \in \mathbb{R}$, with $\phi(t)=1, \Psi(\|x\|)=5$. It is easy to verify that $M>0.775057$. Then, by Theorem 5, problems (20) and (21) with $F(t, x)$ given by (23) has at least one solution on $J$.

(c) Consider the multivalued map $F: J \times \mathbb{R} \rightarrow \mathcal{P}(\mathbb{R})$ given by

$$
x \rightarrow F(t, x)=\left[0,(t+1) \sin x+\frac{2}{3}\right] .
$$

Then we have

$$
\sup \{|u|: u \in F(t, x)\} \leq(t+1)+\frac{2}{3}
$$

and

$$
H_{d}(F(t, x), F(t, z)) \leq(t+1)|x-z| .
$$

Let $\mu(t)=t+1$. Then $H_{d}(F(t, x), F(t, z)) \leq m(t)|x-z|$, and $\|\mu\|=2$. By Theorem 7 , the problem (20)-(21) with the $F(t, x)$ given by (24) has at least one solution on $J$ since $\Lambda\|\mu\| \approx 0.310023<1$. 


\section{Conclusions}

In this paper, we have presented the existence-criteria for solutions of arbitrary order fractional differential equations and inclusions, supplemented with integro-multistrip-multi-point boundary conditions. For obtaining the existence and uniqueness results for the single valued problem, we applied fixed-point theorems due to Krasnoselskii and Banach. The inclusions problem is investigated for convex as well as non-convex valued cases and the obtained results rely on Bohnenblust-Karlin fixed point theorem, Martelli fixed point theorem, Leray-Schauder nonlinear alternative, and Covitz-Nadler fixed point theorem. Our results are not only new in the given setting, but also yield some new ones as special cases. For example, we can obtain the results for integro-multistrip boundary conditions by fixing $\gamma_{j}=0, \forall j=1, \ldots, q$, while the results for integro-multipoint boundary conditions follow when we take $\beta_{i-1}=0, \forall i=2, \ldots, p$. Our results reduce to the ones for "zero" average value condition $\left(\int_{0}^{1} u(s) d s=0\right)$ by taking all $\gamma_{j}=0, \forall j=1, \ldots, q$ and $\beta_{i-1}=0, \forall i=2, \ldots, p$.

Author Contributions: Conceptualization, S.K.N. and B.A.; methodology, S.K.N. and A.A.; validation, S.K.N., B.A. and A.A.; formal analysis, B.A. and S.K.N.; writing—original draft preparation, S.K.N., A.A.; writing一review and editing, B.A. All authors have read and agreed to the published version of the manuscript.

Funding: This research received no external funding.

Conflicts of Interest: The authors declare no conflict of interest.

\section{References}

1. Carvalho, A.; Pinto, C.M.A. A delay fractional order model for the co-infection of malaria and HIV / AIDS. Int. J. Dyn. Control 2017, 5, 168-186. [CrossRef]

2. Ding, Y.; Wang, Z.; Ye, H. Optimal control of a fractional-order HIV-immune system with memory. IEEE Trans. Contr. Syst. Technol. 2012, 20, 763-769. [CrossRef]

3. $\mathrm{Xu}, \mathrm{Y}$.; Li, W. Finite-time synchronization of fractional-order complex-valued coupled systems. Physica A 2020, 549, 123903. [CrossRef]

4. Xu, Y.; Li, Y.; Li, W. Adaptive finite-time synchronization control for fractional-order complex-valued dynamical networks with multiple weights. Commun. Nonlinear Sci. Numer. Simul. 2020, 85, 105239. [CrossRef]

5. Mainardi, F. Fractional calculus: Some basic problems in continuum and statistical mechanis. In Fractals and Fractional Calculus in Continuum Mechanics; Carpinteri, A., Mainardi, F., Eds.; Springer: Wien, NY, USA, 1997; pp. 291-348.

6. Fallahgoul, H.A.; Focardi, S.M.; Fabozzi, F.J. Fractional Calculus and Fractional Processes with Applications to Financial Economics, Theory and Application; Elsevier/Academic Press: London, UK, 2017.

7. Kilbas, A.A.; Srivastava, H.M.; Trujillo, J.J. Theory and Applications of Fractional Differential Equations; North-Holland Mathematics Studies, 204; Elsevier Science B.V.: Amsterdam, The Netherlands, 2006.

8. Ahmad, B.; Alsaedi, A.; Ntouyas, S.K.; Tariboon, J. Hadamard-type Fractional Differential Equations, Inclusions and Inequalities; Springer: Cham, Switzerland, 2017.

9. Bai, Z.B.; Sun, W. Existence and multiplicity of positive solutions for singular fractional boundary value problems. Comput. Math. Appl. 2012, 63, 1369-1381. [CrossRef]

10. Rocha, P.; Urciuolo, M. Fractional type integral operators of variable order. Rev. Un. Mat. Argentina 2017, 58, 281-296.

11. Liu, J.; Zhao, K. Existence of mild solution for a class of coupled systems of neutral fractional integro-differential equations with infinite delay in Banach space. Adv. Differ. Equ. 2019, 2019, 284. [CrossRef]

12. Henderson, J.; Luca, R.; Tudorache, A. On a system of fractional differential equations with coupled integral boundary conditions. Fract. Calc. Appl. Anal. 2015, 18, 361-386. [CrossRef]

13. Wang, Y.; Liang, S.; Wang, Q. Existence results for fractional differential equations with integral and multi-point boundary conditions. Bound. Value Probl. 2018, 2018, 4. [CrossRef]

14. Ahmad, B.; Alsaedi, A.; Salem, S.; Ntouyas, S.K. Fractional differential equation involving mixed nonlinearities with nonlocal multi-point and Riemann-Stieltjes integral-multi-strip conditions. Fractal Fract. 2019, 3, 34. [CrossRef] 
15. Ming, Z.; Zhang, G.; Li, H. Positive solutions of a derivative dependent second-order problem subject to Stieltjes integral boundary conditions. Electron. J. Qual. Theory Differ. Equ. 2019, 2019, 98. [CrossRef]

16. Alsaedi, A.; Ahmad, B.; Aljoudi, S.; Ntouyas, S.K. A study of a fully coupled two-parameter system of sequential fractional integro-differential equations with nonlocal integro-multipoint boundary conditions. Acta Math. Sci. Ser. B 2019, 39, 927-944. [CrossRef]

17. Kisielewicz, M. Stochastic Differential Inclusions and Applications; Springer: New York, NY, USA, 2013.

18. Danca, M.F. Synchronization of piecewise continuous systems of fractional order. Nonlinear Dyn. 2014, 78, 2065-2084. [CrossRef]

19. Korda, M.; Henrion, D.; Jones, C.N. Convex computation of the maximum controlled invariant set for polynomial control systems. SIAM J. Control Optim. 2014, 52, 2944-2969. [CrossRef]

20. Bastien, J. Study of a driven and braked wheel using maximal monotone differential inclusions: Applications to the nonlinear dynamics of wheeled vehicles. Arch. Appl. Mech. 2014, 84, 851-880. [CrossRef]

21. Wang, Y.; Liang, T. Mild solutions to the time fractional Navier-Stokes delay differential inclusions. Discret. Contin. Dyn. Syst. Ser. B 2019, 24, 3713-3740. [CrossRef]

22. Kamenskii, M.; Obukhovskii, V.; Petrosyan, G.; Yao, J.-C. On semilinear fractional order differential inclusions in Banach spaces. Fixed Point Theory 2017, 18, 269-291. [CrossRef]

23. Aissani, K.; Benchohra, M.; Darwish, M.A. Semilinear fractional order integro-differential inclusions with infinite delay. Georgian Math. J. 2018, 25, 317-327. [CrossRef]

24. Abbas, S.; Benchohra, M.; Graef, J.R. Coupled systems of Hilfer fractional differential inclusions in Banach spaces. Commun. Pure Appl. Anal. 2018, 17, 2479-2493. [CrossRef]

25. Ahmad, B.; Ntouyas, S.K.; Alsaedi, A. Coupled systems of fractional differential inclusions with coupled boundary conditions. Electron. J. Differ. Equ. 2019, 2019, 69.

26. Ntouyas, S.K.; Al-Sulami, H.H. A study of coupled systems of mixed order fractional differential equations and inclusions with coupled integral fractional boundary conditions. Adv. Differ. Equ. 2020, 2020, 73. [CrossRef]

27. Cheng, Y.; Agarwal, R.P.; O’Regan, D. Existence and controllability for nonlinear fractional differential inclusions with nonlocal boundary conditions and time-varying delay. Fract. Calc. Appl. Anal. 2018, 21, 960-980. [CrossRef]

28. Cernea, A. On some fractional integro-differential inclusions with nonlocal multi-point boundary conditions. Fract. Differ. Calc. 2019, 9, 139-148. [CrossRef]

29. Ntouyas, S.K.; Alsaedi, A.; Ahmed, B. Existence theorems for mixed Riemann-Liouville and Caputo fractional differential equations and inclusions with nonlocal fractional integro-differential boundary conditions. Fractal Fract. 2019, 3, 21. [CrossRef]

30. Benchohra, M.; Hamani, S.; Zhou, Y. Oscillation and nonoscillation for Caputo-Hadamard impulsive fractional differential inclusions. Adv. Differ. Equ. 2019, 2019, 74. [CrossRef]

31. Yue, Y.; Tian, Y.; Bai, Z. Infinitely many nonnegative solutions for a fractional differential inclusion with oscillatory potential. Appl. Math. Lett. 2019, 88, 64-72. [CrossRef]

32. Ahmad, B.; Ntouyas, S.K.; Tariboon, J. On inclusion problems involving Caputo and Hadamard fractional derivatives. Acta Math. Univ. Comenian. 2020, 89, 169-183.

33. Qarout, D.; Ahmad, B.; Alsaedi, A. Existence theorems for semi-linear Caputo fractional differential equations with nonlocal discrete and integral boundary conditions. Fract. Calc. Appl. Anal. 2015, 19, 463-479. [CrossRef]

34. Krasnoselskii, M.A. Two remarks on the method of successive approximations. Uspekhi Mat. Nauk 1955, 10, 123-127.

35. Aubin, J.-P.; Cellina, A. Differential Inclusions. Set-Valued Maps and Viability Theory; Springer: Berlin, Germany, 1984.

36. Castaing, C.; Valadier, M. Convex Analysis and Measurable Multifunctions; Lecture Notes in Mathematics 580; Springer: Berlin/Heidelberg, Germany; New York, NY, USA, 1977.

37. Deimling, K. Multivalued Differential Equations; De Gruyter: Berlin, Germany, 1992.

38. Bohnenblust, H.F.; Karlin, S. On a theorem of Ville. In Contributions to the Theory of Games; Princeton University Press: Princeton, NJ, USA, 1950; Volume I, pp. 155-160.

39. Lasota, A.; Opial, Z. An application of the Kakutani-Ky Fan theorem in the theory of ordinary differential equations. Bull. Acad. Pol. Sci. Ser. Sci. Math. Astronom. Phys. 1965, 13, 781-786.

40. Martelli, M. A Rothe's theorem for non compact acyclic-valued maps. Boll. Un. Mat. Ital. 1975, 4, 70-76. 
41. Granas, A.; Dugundji, J. Fixed Point Theory; Springer: New York, NY, USA, 2005.

42. Bressan, A.; Colombo, G. Extensions and selections of maps with decomposable values. Stud. Math. 1988, 90, 69-86. [CrossRef]

43. Frigon, M. Théorèmes d'existence de solutions d'inclusions différentielles. In Topological Methods in Differential Equations and Inclusions; Granas, A., Frigon, M., Eds.; NATO ASI Series C; Kluwer Academic Publishers: Dordrecht, The Netherlands, 1995; Volume 472, pp. 51-87.

44. Covitz, H.; Nadler, S.B., Jr. Multivalued contraction mappings in generalized metric spaces. Isr. J. Math. 1970, 8, 5-11. [CrossRef]

(C) 2020 by the authors. Licensee MDPI, Basel, Switzerland. This article is an open access article distributed under the terms and conditions of the Creative Commons Attribution (CC BY) license (http:/ / creativecommons.org/licenses/by/4.0/). 